\title{
COMPARAÇÃO ENTRE OS MÉTODOS DO CILINDRO E DO TORRÃO NA DETERMINAÇÃO DA POROSIDADE E DA DENSIDADE DO SOLO
}

\author{
COMPARISON BETWEEN THE CORE AND CLOD METHODS FOR THE DETERMINATION \\ OF SOIL POROSITY AND BULK DENSITY
}

\author{
Vanderlei Rodrigues da Silva ${ }^{1}$ Dalvan José Reinert ${ }^{2}$ José Miguel Reichert ${ }^{2}$
}

\section{RESUMO}

\begin{abstract}
O objetivo deste estudo foi comparar e estabelecer relações entre a densidade do solo, a porosidade total, macro e microporosidade determinados por dois métodos (cilindro volumétrico tipo Uhland e o método do torrão), em sistema plantio direto e preparo convencional de solo, com e sem pastejo animal. Coletaram-se amostras de um argissolo vermelho distrófico arênico, textura superficial franca, localizado em Santa Maria, $R S$. Os valores de densidade do solo determinados pelo método do torrão foram maiores do que aqueles determinados pelo método do cilindro. A macroporosidade foi o parâmetro do solo que apresenta as maiores variações entre os dois métodos estudados. Assim, deve-se evitar comparar sistemas de manejo, de estudos nos quais foram empregados distintos métodos.
\end{abstract}

Palavras chave: manejo do solo, porosidade e densidade do solo, métodos físicos.

\section{SUMMARY}

The objective of this study was to compare and to establish relations between bulk density, total porosity, macro and microporosity values determined by the Uhland core method and by the clod method, for no tillage and conventional tillage. with and without grazing. Soil samples were collected from a Hapludalf, with a loam surface texture, located in Santa Maria, in Brazil. The bulk density values obtained by the clod method were greater than those obtained by the Uhland core method. The macroporosity was the soil parameter with the greatest variations when comparing the two methods. Thus, comparison it should be avoided to compare management systems, of studies where different methods were used.

Key works: soil management, soil porosity and bulk density, physical methods.
As propriedades físicas de um solo têm influência direta no desenvolvimento radicular da cultura e, conseqüientemente, na sua produtividade. Entre essas propriedades, a densidade do solo (ds) é um parâmetro que serve como índice de compactação do solo (BOWEN, 1981; TOMPSON et al., 1987) e é muito usada na avaliação do estado estrutural dos solos (HAKANSSON \& VOORHEES, 1997). O método do cilindro (MC) é considerado como o método padrão de amostragem para avaliação da porosidade total $(\mathrm{Pt})$ e da ds. Esse método é criticado por provocar uma possível compactação do solo pelo atrito cilindro-solo durante a penetração do mesmo no solo, principalmente quando o solo estiver com umidade acima do ponto de friabilidade, ou de fraturar ou cisalhar o solo quando o mesmo estiver seco (BLAKE \& HARTGE, 1986). O método do torrão (MT) apresenta a desvantagem de poder segregar a amostra pela ocasião da coleta dos torrões em solos que foram mobilizados e não completamente reconsolidados, desconsiderando os macroporos existentes entre os torrões. O MT é bastante útil em solos pedregosos, ou quando o solo estiver muito seco que impeça a penetração do cilindro no solo.

Os valores de ds determinados pelo MT são geralmente maiores que os valores determinados por outros métodos (BLAKE \& HARTGE, 1986; VANREMORTEL \& SHIELDS, 1993). Para solos mobilizados, o MC seria o indicado por apresentar resultados mais confiáveis. Porém, ainda há necessi-

\footnotetext{
${ }^{1}$ Engenheiro Agrônomo, Mestre, doutorando do Programa de Pós-graduação em Agronomia, Universidade Federal de Santa Maria (UFSM), CEP 97105-900, Santa Maria, RS. E-mail: a9760360@alunop.ufsm.br. Autor para correspondência.

${ }^{2}$ Engenheiro Agrônomo, PhD, Professor titular do Departamento de Solos, Centro de Ciências Rurais, UFSM, Santa Maria, RS. Bolsista do CNPq. 
dade de maior investigação das diferenças e do comportamento desses dois métodos, principalmente, para diferentes tipos de solos e manejo. O objetivo deste estudo foi comparar e estabelecer relações entre os valores de ds, Pt, macro (Mac) e microporosidade (Mic) medidos pelo MT e MC, em diversas situações de manejo do solo e pastejo animal.

As amostras para determinação da Pt e da ds foram coletadas na área experimental do Departamento de Zootecnia, UFSM, num argissolo vermelho distrófico arênico, textura superficial franca, com $0,21 \mathrm{~kg} \mathrm{~kg}^{-1}$ de argila; $0,43 \mathrm{~kg} \mathrm{~kg}^{-1}$ de silte e $0,02 \mathrm{~kg} \mathrm{~kg}^{-1}$ de carbono orgânico. A área experimental vinha sendo conduzida sob preparo convencional de solo para a implantação da pastagem de inverno composta por aveia (Avena strigosa Schreb) + azevém (Lolium multiflorum L.) e para a semeadura do milho. A partir de 1993 até 1996, a pastagem e o milho foram semeados sob sistema plantio direto em parte da área, sendo que o restante continuou em sistema convencional de preparo do solo. Cercou-se uma área de $2500 \mathrm{~m}^{2}$ para servir como testemunha, não recebendo pisoteio do gado, mas manteve-se os tratamentos de preparo do solo. $\mathrm{Na}$ área pastejada, o número de animais foi variável em função da produção de matéria seca da pastagem, permanecendo em torno de $1,0 \mathrm{Mg} \mathrm{ha}^{-1}$ de peso vivo. Os tratamentos foram: 1) plantio direto de milho e sem pastejo no inverno; 2) plantio direto de milho com pastejo contínuo durante o inverno; 3 ) preparo convencional antes da semeadura do milho sem pastejo no inverno e; 4) preparo convencional antes da semeadura do milho com pastejo contínuo no inverno. $\mathrm{O}$ delineamento experimental foi de blocos ao acaso, com quatro repetições de campo. Maiores detalhes da instalação do experimento encontra-se em BASSANI (1996).

As amostras para avaliações das propriedades físicas do solo foram coletadas em três épocas: antes do pastejo; logo após a retirada do gado e após a colheita do milho, nos três anos de condução do experimento. Para o $\mathrm{MC}$ com altura e diâmetro = $0,076 \mathrm{~m}$, foram coletados quatro cilindros por parcela, na profundidade de 0 a $0,1 \mathrm{~m}$. Os cilindros foram saturados, pesados e levados à mesa de tensão no potencial de $6 \mathrm{kPa}$ para determinar a Mic. Após, o solo de cada cilindro foi seco em estufa a $105^{\circ} \mathrm{C}$. Com esses dados foram calculados a Mic, a ds, a Pt por saturação (massa de água quando a amostra estava saturada) e por cálculo pela fórmula $\mathrm{Pt}=\left(1-\frac{\mathrm{ds}}{\mathrm{dp}}\right)$, onde: $\mathrm{Pt}=$ porosidade total $; \mathrm{ds}=$ densidade do solo e $\mathrm{dp}=$ densidade de partícula.
Para o MT, foram coletados três torrões em cada ponto de coleta do cilindro, compondo uma média para correlacionar com os dados do cilindro. As amostras foram preparadas, pesadas e após mergulhadas em parafina fundida para impermeabilização. Em seguida, o torrão impermeabilizado com parafina foi fixado na parte inferior da balança com o auxílio de uma cesta de arame, pesado no ar e pesado imerso em água com temperatura conhecida. Após essas pesagens, o torrão foi partido em duas metades, sendo que em uma foi retirada a parafina e levada à estufa para determinação da umidade gravimétrica. A outra metade foi saturada e colocada em mesa de tensão no potencial de $6 \mathrm{kPa}$, tendo o cuidado de fazer um pequeno orifício na parte superior do torrão, posteriormente foi secado a $105^{\circ} \mathrm{C}$. Com os dados obtidos, calcularam-se a ds, Mic, Mac e Pt.

No MT, os valores de ds foram maiores e os de Pt menores do que aqueles obtidos pelo MC (Figura 1a, 1b). Esse fato pode estar associado à penetração da parafina em macroporos e fendas existentes no torrão, ou à perda de macroporos e segregação no momento da coleta dos torrões acarretando maiores valores de ds (BLAKE \& HARTGE, 1986) e menores valores de Mac. VANREMORTEL \& SHIELDS (1993) também encontraram valores maiores de ds para o MT do que para o MC. Porém, o coeficiente de determinação $\left(r^{2}\right)$ obtido da relação entre os valores de ds obtidos por esses dois métodos foi bastante alto $(0,98)$, enquanto que para o presente estudo, o $\mathrm{r}^{2}$, apesar de altamente significativo, foi de apenas 0,40, indicando grande dispersão dos dados. Por exemplo, quando se considera uma densidade do solo, determinada pelo $\mathrm{MC}$, de $1,30 \mathrm{Mg} \mathrm{m}^{-3}$, observam-se valores de ds determinado pelo MT variando de 1,30 a $1,60 \mathrm{Mg} \mathrm{m}^{-3}$ (Figura 1a).

Quando comparado com o MC, o MT apresentou os maiores valores de Mic e de ds e, conseqüentemente, menores de $\mathrm{Pt}$ (Tabela 1). Porém, o coeficiente de variação $(\mathrm{CV})$ foi menor para o parâmetro ds e maior para os outros parâmetros do solo. Analisando os CVs entre os dois métodos, verifica-se que a Mac é o parâmetro que apresentou o maior $\mathrm{CV}, 30,1 \%$ no $\mathrm{MC}$ e $74,5 \%$ no MT.

Quando se separam os sistemas de manejo e correlacionam-se todas as épocas de coleta de dados, há melhor correlação entre os dados obtidos para o preparo convencional de solo do que entre os dados obtidos somente no sistema plantio direto (Figura 1c, 1d). Essa constatação não confirma a hipótese de que o MC apresenta resultados

Ciência Rural, v. 30, n. 6, 2000. 


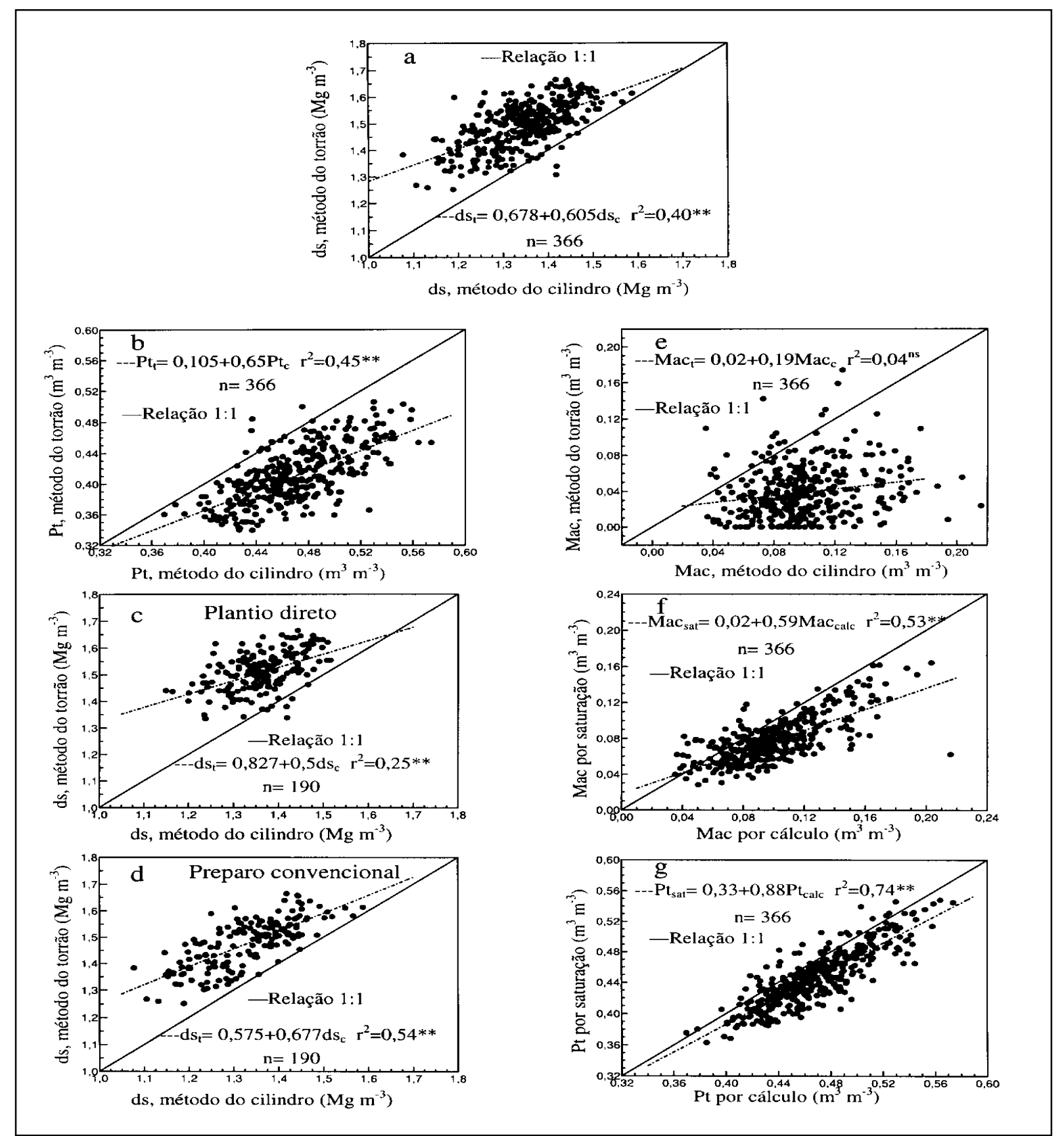

Figura 1 - Relação entre o método do torrão e o método do cilindro na determinação da densidade do solo (a), porosidade total (b), densidade do solo para sistema plantio direto (c) e preparo convencional (d), macroporosidade (e), relação entre a macroporosidade determinada por saturação e por cálculo (f) e a porosidade total determinada por saturação e por cálculo $(\mathrm{g})$.

mais confiáveis em solos mobilizados, quando comparados aos resultados obtidos pelo MT. A Mac, determinada por cálculo, foi maior quando medida pelo MC, não apresentando correlação significativa com os dados obtidos pelo MT (Figura 1e).

Para o MT, a Pt foi determinada também por saturação. A Mic foi considerada a quantidade de água retida no solo na tensão de $6 \mathrm{kPa}$. A diferen- ça entre a Pt por saturação e a Mic, corresponde a Mac por saturação. Os valores de Pt e Mac determinados por saturação foram menores quando comparados com os valores obtidos por cálculo (Figura 1f, $1 \mathrm{~g}$ ), devido à dificuldade em saturar-se completamente a amostra, ou à perda de água antes da pesagem, principalmente dos bioporos. 
Tabela 1 - Valores médios, mínimos, máximos, desvio padrão $(\sigma)$ e coeficiente de variação $(\mathrm{CV})$ de alguns atributos do solo obtidos pelo método do cilindro e pelo método do torrão.

\begin{tabular}{|c|c|c|c|c|c|c|c|c|c|c|}
\hline \multirow{2}{*}{ Atributo } & \multicolumn{5}{|c|}{-------------------- Método do Cilindro ------------------ } & \multicolumn{5}{|c|}{ 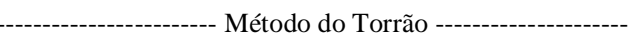 } \\
\hline & média & mínimo & máximo & $\sigma$ & $\mathrm{CV}(\%)$ & média & mínimo & máximo & $\sigma$ & CV (\%) \\
\hline $\mathrm{Ds}\left(\mathrm{Mg} \mathrm{m}^{-3}\right)$ & 1,35 & 1,08 & 1,59 & 0,087 & 6,4 & 1,49 & 1,25 & 1,66 & 0,083 & 5,5 \\
\hline $\operatorname{Pt}\left(\mathrm{cm}^{3} \mathrm{~cm}^{-3}\right)$ & 0,47 & 0,37 & 0,57 & 0,036 & 7,7 & 0,41 & 0,34 & 0,51 & 0,035 & 8,6 \\
\hline $\operatorname{Mic}\left(\mathrm{cm}^{3} / \mathrm{cm}^{-3}\right)$ & 0,37 & 0,21 & 0,47 & 0,031 & 8,5 & 0,38 & 0,31 & 0,58 & 0,036 & 9,5 \\
\hline $\operatorname{Mac}_{\text {calc }}\left(\mathrm{cm}^{3} \mathrm{~cm}^{-3}\right)$ & 0,10 & 0,04 & 0,22 & 0,030 & 30,1 & 0,04 & 0,00 & 0,17 & 0,029 & 74,5 \\
\hline $\operatorname{Mac}_{\mathrm{sat}}\left(\mathrm{cm}^{3} \mathrm{~cm}^{-3}\right)$ & 0,08 & 0,03 & 0,16 & 0,024 & 31,5 & $---{ }^{(1)}$ & ---- & --- & ---- & ---- \\
\hline
\end{tabular}

$\mathrm{ds}=$ densidade do solo; $\mathrm{Pt}=$ porosidade total; $\mathrm{Mic}=$ microporosidade; Mac $_{\mathrm{calc}}=$ macroporosidade determinada por cálculo; $\mathrm{Mac}_{\text {sat }}=$ macroporosidade estimada pela saturação.

(1) Valores não estimados.

Em conclusão, deve-se evitar comparações entre sistemas de manejo que utilizaram métodos distintos na determinação da densidade do solo, porosidade total, macro e microporosidade. Os resultados de porosidade total, macro e microporosidade apresentam menor variação quando determinados pelo método do cilindro.

\section{REFERÊNCIAS BIBLIOGRÁFICAS}

BASSANI, H.J. Propriedades físicas induzidas pelo plantio direto e convencional em área pastejada e não pastejada. Santa Maria, RS, 1996. 90p. Dissertação (Mestrado em Agronomia/Biodinâmica de Solos) - Programa de Pósgraduação em Agronomia, Universidade Federal de Santa Maria, 1996.

BLAKE, G.R., HARTGE, K.H. Bulk density. In: KLUTE, A, (ed). Methods of soil analysis. Physical and mineralogical methods. Madison : ASA, 1986. p.363-375.

BOWEN, H.D. Alleviating mechanical impedance. In: ARKIN, G.F., TAYLOR, H.M., (eds). Modifying the root environment to reduce crop stress. St. Joseph : American Society of Agricultural Engineers, 1981. p.21-57.

HAKANSSON, I., VOORHEES, W.B. Soil compaction. In: LAL, R., BLUM, W.H., VALENTIN, C., et al. (eds). Methods for assessment of soil degradation. Boca Raton: Lewis, 1997. p.167-179.

THOMPSON, P.J., JANSEN, I.J., HOOKS, C.L. Penetrometer resistance and bulk density as parameters for predicting root system performance in mine soils. Soil Science Society of America Journal, Madison, v.51, p.1288-1293, 1987.

VANREMORTEL, R.D., SHIELDS, D.A. Comparison of clod and core methods for determination of soil bulk-density. Communications in Soil Science and Plant Analysis, v.24, p.2517-2528, 1993 .

Ciência Rural, v. 30, n. 6, 2000. 